\title{
Benefits of Concurrent Aerobic-Resistance Interval Exercise in Patients with Chronic Obstructive Pulmonary Disease
}

\author{
Carolina Aristizábal ${ }^{1}$, Oscar Ortiz ${ }^{1}$, Emily J. Walsh ${ }^{1,3}$, Mario Leone $^{2,4}$ and Alain S. Comtois ${ }^{1 *}$ \\ ${ }^{1}$ Département des Sciences de l'activité Physique, Université du Québec à Montréal, Canada \\ ${ }^{2}$ Département des Sciences de la Santé, Université du Québec à Chicoutimi, Canada \\ ${ }^{3}$ Loyola University Chicago Stritch School of Medicine, USA \\ ${ }^{4}$ Faculté de médecine, Université de Sherbrooke, Canada
}

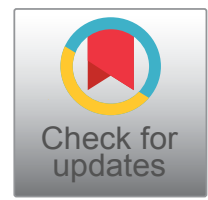

*Corresponding author: Alain S. Comtois, PhD, Département des Sciences de l'activité Physique, Université du Québec à Montréal, Montreal, Québec, H3C $3 P 8$,, Canada

\begin{abstract}
Background: Physical training of lower leg skeletal muscle has been shown to increase exercise tolerance in Chronic Obstructive Pulmonary Disease (COPD) patients. The objective of this study was to measure the effect of concurrent aerobic-resistance interval exercise on lower leg muscle function and local muscle tissue oxygenation in the vastus lateralis muscle (VLM) of COPD patients.
\end{abstract}

Methods: Peripheral muscle oxygenation in the VLM was measured using Near Infrared Spectrometry (NIRS) during the six- minute walk test (6MWT) in 15 COPD patients: Experimental (EXP., $\mathrm{n}=9$ ) and control (CTL., $\mathrm{n}=6$ ). Both groups trained for 3 weeks ( 1 hour/day and 5 days/ week). Training sessions consisted of upper body strength exercises for 20 minutes. Next, the CTL. Group performed 30 minutes of aerobic exercise (motorized treadmill or stationary bike), while the EXP. group completed 30 minutes of aerobic-resistance (concurrent training) interval exercise (non-motorized treadmill). Pre and post-intervention NIRS measurements included oxyhemoglobin $\left(\mathrm{O}_{2} \mathrm{Hb}\right)$, deoxyhemoglobin $(\mathrm{HHb})$, hemoglobin difference (HbDif), total hemoglobin $(\mathrm{tHb})$, and tissue saturation index (TSI). Systemic arterial oxygen saturation $\left(\mathrm{SPO}_{2}\right)$, heart rate and the rating of perceived exertion with the modified Borg scale were also measured pre and post-intervention during the 6MWT.

Results: All patients were able to complete three weeks of training. A significant increase in the 6MWT (51.4\%, p $<0.05$ ) distance was noted in the EXP. group and in the VLM HbDiff $(69 \%, p<0.05)$. No significant differences were observed in the other muscle oxygenation variables.
Conclusion: Concurrent aerobic-resistance interval exercise increases the total distance covered at the 6MWT (increased functional capacity) and muscle oxygen extraction.

\section{Keywords}

COPD, Muscle oxygenation, Interval exercise, NIRS, Muscle oxygen kinetic, Concurrent aerobic-resistance exercise

\begin{abstract}
Abbreviations
VLM: Vastus Lateralis Muscle; NIRS: Near Infrared Spectrometry; 6MWT: Six-Minute Walk Test; $\mathrm{O}_{2} \mathrm{Hb}$ : Oxyhemoglobin; Hhb: Deoxyhemoglobin; Hbdif Hemoglobin Difference; Thb: Total Hemoglobin; TSI: Tissue Saturation Index; $\% \mathrm{SpO}_{2}$ : Systemic Oxygen Saturation
\end{abstract}

\section{Objectives}

Chronic Obstructive Pulmonary Disease (COPD) is a debilitating condition affecting the lives of millions people world-wide. COPD affects not only the respiratory system, but also peripheral skeletal muscles through type I and type IIA muscle fiber atrophy that leads to impairment in both muscle oxidative capacity and muscle strength [1]. Physical training has been shown to improve muscle function and structure in COPD patients [2]. Physical training improves muscle fiber oxidative capacity, increases the cross-sectional area of muscle fibers type $I$ and Ila, and reduces submaximal exercise lactate concentration [3].

Citation: Aristizábal C, Ortiz O, Walsh EJ, Leone M, Comtois AS (2021) Benefits of Concurrent AerobicResistance Interval Exercise in Patients with Chronic Obstructive Pulmonary Disease. Int J Sports Exerc Med 7:209. doi.org/10.23937/2469-5718/1510209

Accepted: December 10, 2021; Published: December 12, 2021

Copyright: (C) 2021 Aristizábal C, et al. This is an open-access article distributed under the terms of the Creative Commons Attribution License, which permits unrestricted use, distribution, and reproduction in any medium, provided the original author and source are credited. 
It is well established that pulmonary rehabilitation programs are important in the treatment of patients with COPD because they increase exercise tolerance, reduce symptoms of dyspnea and improve healthrelated quality of life [4]. There are substantial variations in exercise protocols used in pulmonary rehabilitation programs [5]. One modality that appears promising to offer sustainable high intensity exercise for COPD patients while allowing for short periods of recovery preventing high lactate accumulation is interval exercise [6]. During the training session, patients exercise at high intensity followed by a period of lower intensity or rest. This alternating cycle could be repeated several times. This training modality has been shown to induce less dynamic hyperinflation, and better exercise tolerance than continuous exercise in COPD patients $[7,8]$. Thus, investigators have shown that both aerobic and resistance type training help to alleviate respiratory and peripheral muscle discomfort [9]. However, to the best of our knowledge, no studies have reported the use of concurrent aerobic-resistance as a training modality.

Non-motorized belt driven treadmills involve propelling the treadmill belt while either walking or running [10]. The potential benefit of non-motorized treadmills is that they produce a simultaneous demand of lower leg force production (resistance) and an overall increased aerobic demand. Although recent studies have shown that healthy participants exercising on nonmotorized treadmills improved both muscle force and aerobic capacity, non-motorized treadmills have not been used with COPD patients [11]. The overall objective of this study was to use a non-motorized treadmill as part of a pulmonary rehabilitation program with moderate to severe COPD patients. The specific objective was to measure the effect of concurrent aerobic-resistance exercise on local muscle tissue oxygenation in the VLM and consequently, on functional walking capacity of COPD patients. The hypothesis was that the combined effect of aerobic-resistance exercise would improve VLM oxygenation.

\section{Methods}

\section{Patients}

Twenty-four patients with COPD, referred for inpatient pulmonary rehabilitation at Mount Sinai Hospital, Montreal, were invited to participate in the study. All patients underwent spirometry at the pulmonary function laboratory. Inclusion criteria were men and women aged 50-80, who were hospitalized and part of the rehab program at Mt. Sinai Hospital with a conformed moderate to severe COPD diagnosis, were stable and ambulatory, and had a $<70 \% \frac{F E V_{1}}{V C}<70 \%$ and a FEV1 greater than $30 \%$ but less than $80 \%$ of the predicted value according to the Global Initiative for Obstructive Lung Disease (GOLD) criteria [12].
Exclusion criteria were severe or unstable cardiovascular disease, cognitive impairment, musculoskeletal limitation and neuromuscular disorders. A total of 24 patients were recruited. Nine patients did not complete the training and were excluded from the study. The final sample for analysis was composed of 15 patients.

Upon arrival at Mount Sinai Hospital, each patient received an explanation about the purpose and procedures involved in the study and were able to ask questions before giving their informed written consent. The study received IRB approval (approval number 2013_A_130064) by the ethics committee of the Mount Sinai Hospital and the University of Quebec in Montreal.

\section{Study design}

Patients followed a 3-week multidisciplinary pulmonary rehabilitation program, including exercise training sessions. Before and after the program, all patients retained for the study underwent an anthropometric evaluation (height, weight, leg circumference and skin fold thickness) and a 6MWT that included an assessment of peripheral muscle oxygenation kinetics using NIRS. Measurement details are provided below. The study was designed as a randomized controlled clinical trial. Patients were randomly divided into two groups: The control (CTL.) group had endurance training alone $(n=6)$ and the experimental (EXP.) group received the concurrent training modality (aerobic-resistance, $n=9$ ).

\section{Six-minute walk test procedure}

The 6MWT adhered to the guidelines outlined by the American Thoracic Society [13]. All participants completed the 6MWT. In all patients, before, during and after the 6MWT, dyspnea severity was measured with a modified Borg Scale, while systemic oxygen saturation $\left(\% \mathrm{SpO}_{2}\right)$ and heart ratewere monitored using a finger probe pulse oximeter (MD300 Wrist Pulse Oximeter, $\mathrm{HH}, \mathrm{DE}$ ) and a heart rate monitor (RS800, Polar, Fi). Peripheral tissue muscle oxygenation $\left(\% \mathrm{StO}_{2}\right)$ was measured before, during and after the 6MWT, with a NIRS device (Portamon, Artinis, Medical System, NL).

\section{Muscle oxygenation and arterial oxygenation}

Vastus lateralis $\% \mathrm{StO}_{2}$ was continuously measured with the NIRS device during rest, the 6MWT, and recovery periods. The theory of NIRS has been described in detail elsewhere [14]. The Beer-Lambert law was used to calculate micromolar changes in tissue oxygenation $\left[\left(\mathrm{O}_{2} \mathrm{Hb}\right)\right.$ and $\left.(\mathrm{HHb})\right]$. The changes in concentration of $\mathrm{O}_{2} \mathrm{Hb}, \mathrm{HHb}, \mathrm{tHb}, \mathrm{HbDiff}$, and TSI were measured during the entire 6MWT.

Arterial $\% \mathrm{SpO}_{2}$ was continuously measured during rest, the 6MWT, and recovery periods with a pulse oximeter (MD300 Wrist Pulse Oximeter, HH, DE) using a 
Table 1: Patient characteristics before and after pulmonary rehabilitation programs.

\begin{tabular}{|l|l|l|l|l|l|l|}
\hline \multicolumn{2}{|l}{ PRE-TRAINING } & \multicolumn{3}{l|}{} \\
\hline Patient characteristics & CTL & EXP & $\boldsymbol{p}$ & CTL & EXP \\
\hline Age $(\mathrm{yr})$ & $68.6 \pm 6.8$ & $67.0 \pm 7.1$ & 0.68 & & \\
\hline Weight $(\mathrm{kg})$ & $71.7 \pm 13.0$ & $70.2 \pm 21.1$ & 0.88 & $71.5 \pm 14.5$ & $71.0 \pm 20.9$ & 0.80 \\
\hline Body mass index $\left(\mathrm{kg} / \mathrm{m}^{2}\right)$ & $27.1 \pm 4.5$ & $26.4 \pm 7.0$ & 0.85 & $27.5 \pm 4.6$ & $26.5 \pm 6.7$ & 0.76 \\
\hline Fat mass $(\%)$ & $37.5 \pm 9.1$ & $29.9 \pm 19.9$ & 0.43 & $36.8 \pm 9.5$ & $29.6 \pm 13.3$ & 0.48 \\
\hline Muscle $(\%)$ & $25.2 \pm 3.2$ & $28.9 \pm 7.18$ & 0.85 & $26.0 \pm 3.8$ & $29.4 \pm 6.1$ & 0.36 \\
\hline TPR $(\mathrm{cm})^{*}$ & $46.1 \pm 2.3$ & $42.0 \pm 4.47$ & 0.52 & $48.1 \pm 4.61$ & $43.4 \pm 4.5$ & 0.47 \\
\hline TPL $(\mathrm{cm})^{*}$ & $43.7 \pm 3.5$ & $41.0 \pm 2.92$ & 0.59 & $46.0 \pm 4.9$ & $46.5 \pm 4.8$ & 0.45 \\
\hline
\end{tabular}

Values are presented as group means \pm SD. CTL., Control group; EXP: Experimental group.

*TPR: Thigh Perimeter Right leg, "TPL: Thigh Perimeter Left leg

finger probe attached to either the index or major finger of the participant.

\section{Pulmonary rehabilitation program}

Exercise training in the pulmonary rehabilitation program was either the standard training (CTL. group) typically used at Mount Sinai Hospital (details provided below) or the experimental (EXP.). The exercise training consisted of 5 sessions per week (Monday to Friday) for 3 consecutive weeks for a total number of 15 sessions. Each session lasted 60 minutes and consisted of a 5 minute warm-up followed by 20 minutes of strength exercises for upper body (hand weights or elastic bands), 30 minutes of specific training (aerobic or concurrent aerobic-resistance), and then 5 minutes of cool-down.

For the CTL. group, the exercise training included treadmill walking (initial speed at $1.5 \mathrm{~km} / \mathrm{h}$ increased every week by $0.5 \mathrm{~km} / \mathrm{h}$, and $0^{\circ}$ elevation) or cycling on stationary ergocycles. Pedaling cadence was gradually adjusted according to the patient's tolerance. For the EXP. group, the patients exercised with concurrent interval workouts on a non-motorized treadmill (HiTrainer, Bromont, QC), where the intervals were 30 secs of walking at an intensity of $85-90 \%$ of maximal predicted heart rate followed by a $30 \mathrm{sec}$ rest period (standing still on the treadmill) before beginning the next interval. The number of intervals ranged from 3-5 in the $1^{\text {st }}$ week and for weeks, 2 and 3 progressed to 8 intervals.

\section{Statistical analysis}

All values were reported as mean \pm S.D. The Shapiro-Wilks test was performed to verify the normal distribution of the data. Repeated measures ANOVA was used to test for within and between group effects across time. If significant differences were detected, a Bonferroni adjusted t-test was used to detect the differences between groups. For all analyses, the level of significance was set at $p<0.05$. The statistical analysis of the collected data was performed with Statistical Package for the Social Sciences, version 20 (SPSS Inc., Chicago, IL, USA).

\section{Results}

\section{Physical characteristics}

Both groups were closely matched in gender, age, height, weight, and body mass index (see Table 1). The pulmonary function test revealed moderate to severe air flow limitation, on the basis of global initiative (GOLD) for COPD [12]. After 3 weeks of pulmonary rehabilitation, the 6MWT was significantly increased in both the CTL. and EXP. groups and revealed a significant difference between groups. A greater significant increase in total distance was observed in the EXP. Group [! 6MWT; EXP. $=130.9 \mathrm{~m}(p=0.00) ; C T L .=48.2 \mathrm{~m}(p=0.03)]$. The perception of effort, however, did not show significant changes; although there was a slight decrease postintervention in the EXP. group compared to the CTL. group (intra and intergroup, respectively, ! Borg; EXP. = $-0.56, p=0.44 ; C T L .=0.33, p=0.70$; and EXP. vs. CTL. = $-1.61, p=0.14)$.

\section{Quadriceps oxygenation during the 6-min walk test}

The dynamic behavior of $\mathrm{O}_{2}$ during the 6MWT is shown in Figure 1. This shows that after three weeks of pulmonary rehabilitation, the HbDiff for the CTL. group of $14.07 \pm 9.35 \mu \mathrm{M}$ was significantly different than the EXP. group of $13.72 \pm 7.29 \mu \mathrm{M}(\mathrm{p}=0.004)$. The $\mathrm{O}_{2} \mathrm{Hb}$, however, was not significantly different $(p=0.149)$ between the CTL. group $(25.12 \pm 20.03 \mu \mathrm{M})$ and the EXP. group $(16.48 \pm 16.64 \mu \mathrm{M})$, as was the $\mathrm{HHb}$ (CTL. 38.06 \pm 19.22 vs. EXP. $30.20 \pm 11.27 \mu \mathrm{M}, \mathrm{p}=0.078$ ). Table 2 shows a summary of muscle oxygenation during the 6MWT. The CTL. group showed no significant change in local muscle tissue oxygenation variables, but there was a significant change in the HbDiff. $(p=0.004)$ for the EXP. group. In both groups there were no significant changes in $\mathrm{O}_{2} \mathrm{Hb}, \mathrm{HHb}, \mathrm{tHb}, \mathrm{TSI}$, but a consistent tendency to decrease for the 6MWT with an apparent evident increase in HbDiff.

\section{Heart rate and arterial oxygen saturation}

Following the intervention, the EXP. group showed 


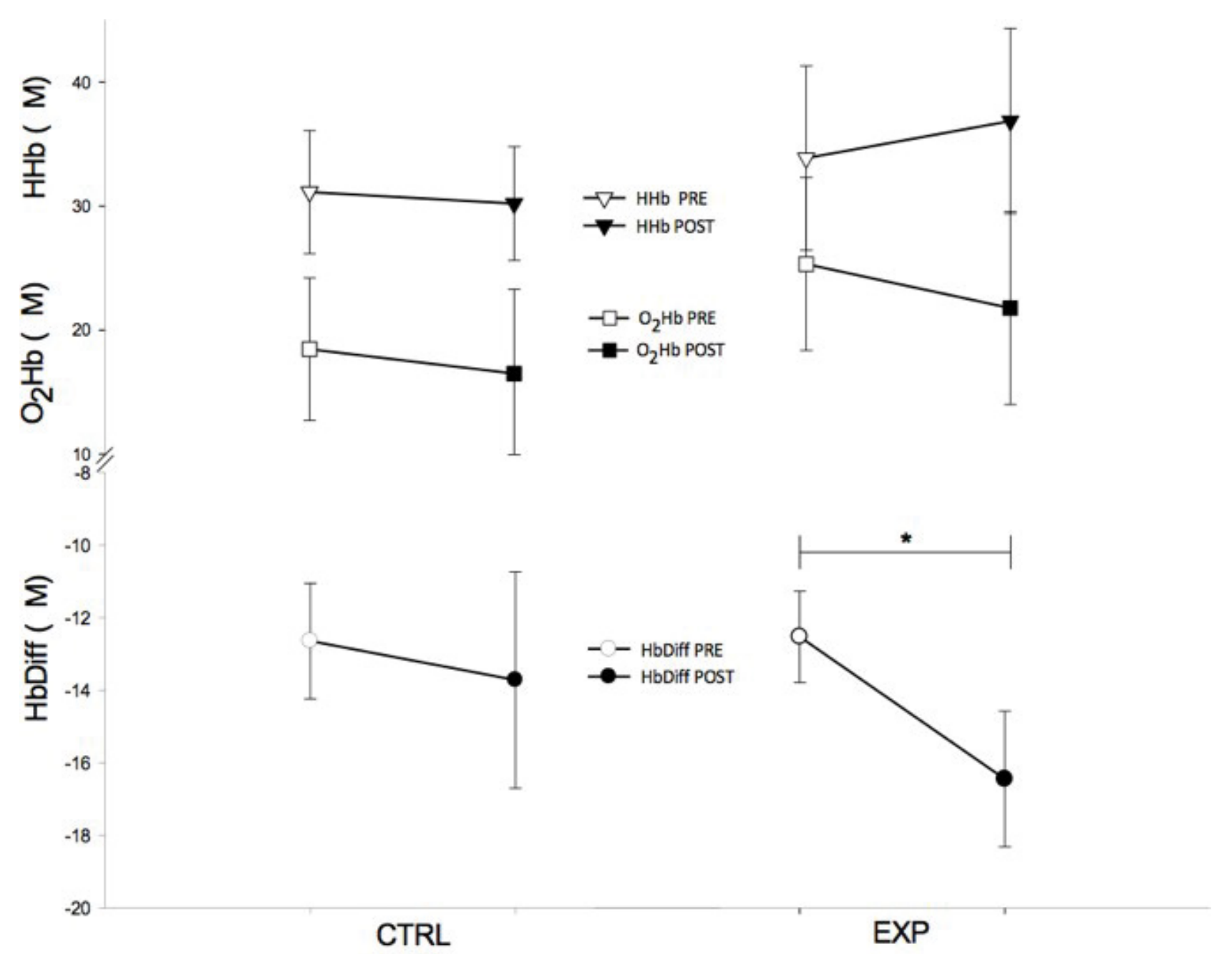

Figure 1: Tissue muscle oxygenation before and after the control (CTL.) and experimental (EXP.) pulmonary rehabilitation program. Top graphs, deoxyhemoglobin $(\mathrm{HHb})$, before $(\square)$ and after $(\boldsymbol{\nabla})$; Middle graphs, oxyhemoglobin $\left(\mathrm{O}_{2} \mathrm{Hb}\right)$ before $(\#)$ and after ( $\mathbf{m})$; and Bottom graphs, hemoglobin difference $(\mathrm{HbDiff})$ calculated by subtracting $\left(\mathrm{O}_{2} \mathrm{Hb}-\mathrm{HHb}\right)$ before (\$) and after $(\cdot)$. All units are expressed in $\mu \mathrm{M} .{ }^{*} \mathrm{p}<0.05$.

Table 2: Leg muscle (vastus lateralis) oxygenation before and after pulmonary rehabilitation programs.

\begin{tabular}{|l|l|l|l|l|l|l|}
\hline \multicolumn{2}{|l}{ CTL } & EXP & \multicolumn{2}{l|}{} \\
\hline Variables & Pre & Post & $\boldsymbol{p}$ & Pre & Post & p \\
\hline $\mathrm{O}_{2} \mathrm{Hb}(\mu \mathrm{M})$ & $18.45 \pm 14.07$ & $16.48 \pm 16.64$ & 0.478 & $28.50 \pm 18.05$ & $25.12 \pm 20.03$ & 0.149 \\
\hline $\mathrm{HHb}(\mu \mathrm{M})$ & $31.12 \pm 12.15$ & $30.20 \pm 11.27$ & 0.586 & $35.49 \pm 19.13$ & $38.06 \pm 19.22$ & 0.078 \\
\hline $\mathrm{HHb}(\mu \mathrm{M})$ & $49.58 \pm 26.00$ & $46.02 \pm 27.11$ & 0.398 & $64.00 \pm 36.60$ & $60.60 \pm 39.08$ & 0.325 \\
\hline $\mathrm{HbDiff}(\mu \mathrm{M})$ & $-12.64 \pm 3.89$ & $-13.72 \pm 7.29$ & 0.652 & $-7.54 \pm 6.27$ & $-14.07 \pm 9.35$ & $0.004^{*}$ \\
\hline TSI $(\%)$ & $63.02 \pm 11.04$ & $60.50 \pm 5.55$ & 0.493 & $61.53 \pm 15.58$ & $59.81 \pm 9.49$ & 0.568 \\
\hline
\end{tabular}

Values are presented as group means \pm SD. "indicates significant difference.

CTL: Control group; EXP: Experimental group; $\mathrm{O}_{2} \mathrm{Hb}$ : Oxyhemoglobin; HHb: deoxyhemoglobin; tHb: Hemoglobin total; TSI: Tissue Saturation Index in the muscle; HbDiff. Hemoglobin Difference; $\mu \mathrm{M}$ : Micromolar/L

a significant increase $(p=0.023)$ for $\mathrm{HR}$, while $\mathrm{SpO}_{2}$ significantly decreased $(p=0.022)$ in Figure 2.

It can be observed that the EXP. group had a greater significant $(p=0.003)$ arterial desaturation postintervention where $\% \mathrm{SpO}_{2}$ for the EXP. group was 87.2 \pm 3.4 when compared to $91.4 \pm 2.3 \%$ for the CTL. group.

\section{Discussion}

The purpose of this study was to evaluate peripheral muscle oxygenation in COPD patients during the 6MWT following a concurrent aerobic resistance interval exercise program. A unique feature of the present study was that oxygen kinetics were continuously measured with NIRS during the 6MWT, before and after the pulmonary rehabilitation program. NIRS has been found to be effective when examining conditions of increased $\mathrm{O}_{2}$ demand in COPD patients [15]. In contrast, several studies in COPD have documented oxygen kinetics 

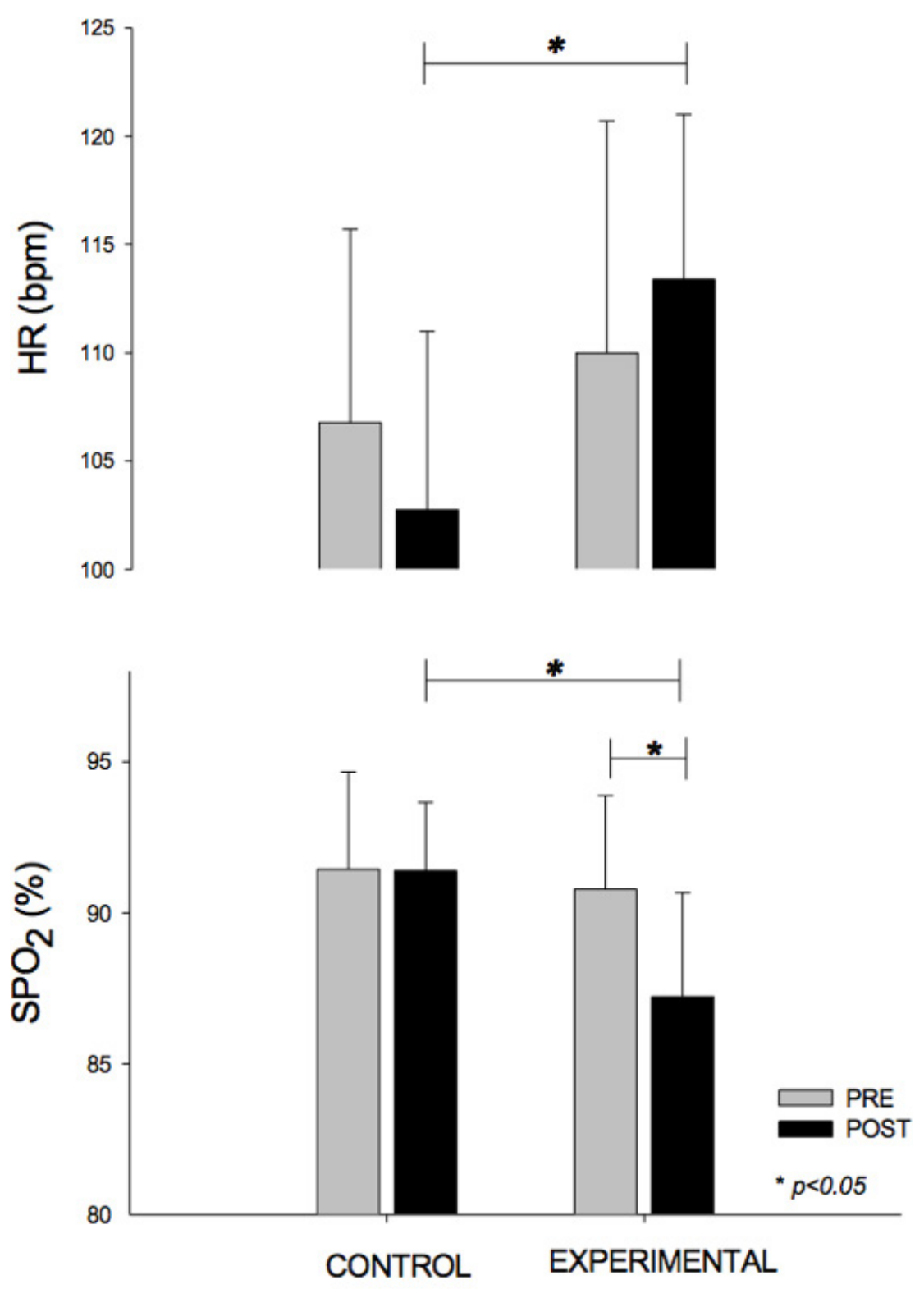

Figure 2: Heart rate (HR) and systemic peripheral oxygen saturation $\left(\% \mathrm{SpO}_{2}\right)$ before (grey) and after (black) the control and experimental pulmonary rehabilitation program. Top graph, $\mathrm{HR}(\mathrm{bpm})$; and Bottom graph, $\% \mathrm{SpO}_{2}$ both at the end of the 6-min walk test $(6 \mathrm{MWT}) .{ }^{*} \mathrm{p}<0.005$.

during exercise or at rest, but not pre and post exercise programs [16-19].

In the current study, it was observed that a 3-week concurrent aerobic-resistance exercise training intervention increased 6MWT distance in COPD patients. The EXP. group showed an average increase of $\sim 130$ meters or $\sim 48 \%$, while the CTL. group increased only by 48 meters or $\sim 17 \%$, after the program. An important outcome was the increase of 131 meters for the EXP. group, which is much greater than the clinically relevant distance of 30.5 meters [20]. There is no doubt that the EXP. group in the current study improved their functional walking capacity. A possible advantage of the procedure is that the progressive resistance on a selfpropelled treadmill (HiTrainer) accelerates leg muscle gains in strength (force) and power.

Puente-Maestu, et al. reported that high intensity training improves muscle oxygen capacity and oxygenation recovery kinetics due to the increased oxidative enzymes in COPD patients [17]. Thus, it is possible that the EXP. patients in the present study improved muscle oxygenation capacity of the lower legs based on the high intensity training they received. The observed improvements were obtained with an exercise program that took place five times a week for three consecutive weeks. This is in contrast to the American Thoracic Society that recommends a minimum of 3 times per week over an 8-week period to reach physiological adaptations and benefits in peripheral skeletal muscles [13]. Importantly, the proposed interval exercise modality in the current study may be an alternative for sites where time constraint is an issue. Nonetheless, further research is necessary to ascertain the efficiency of this type of training in COPD patients. 


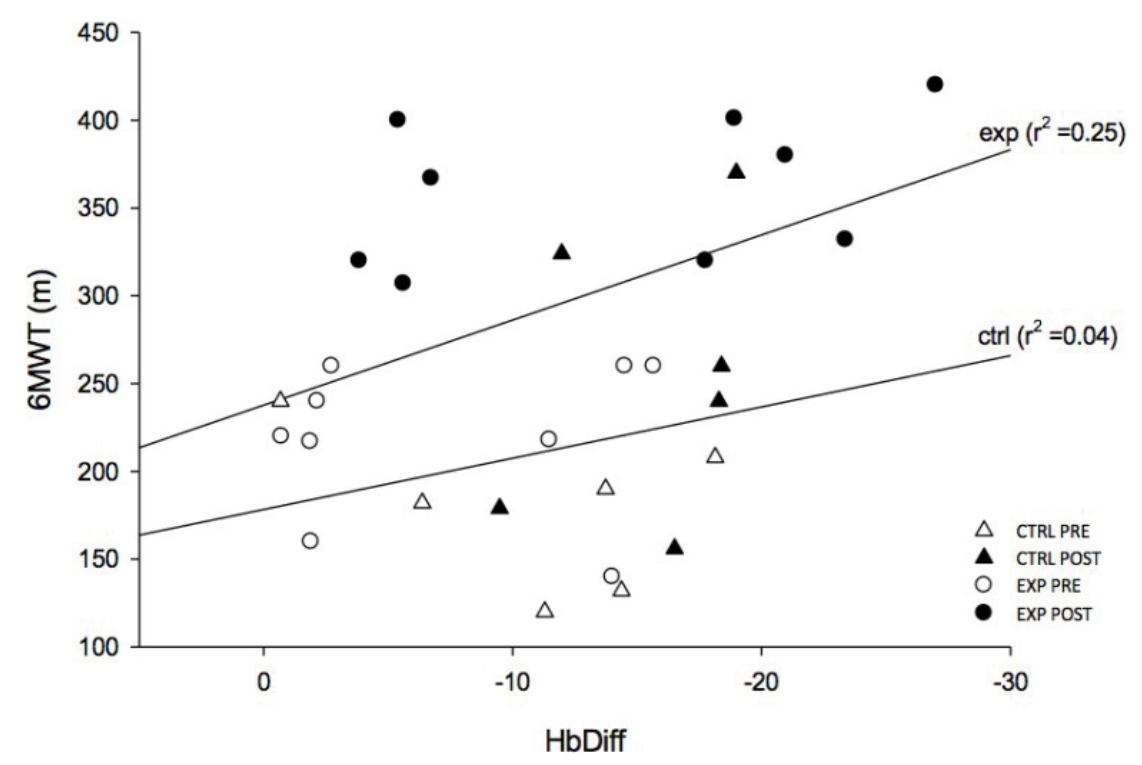

Figure 3: The relationship of total distance walked $(\mathrm{m})$ during the 6 -min walk test (6MWT) as a function of the hemoglobin difference (HbDiff). The empty and filled triangles represent the control (CTL.) group before (! pre) and after ( $\boldsymbol{\Delta}$ post) the intervention whilst the empty and filled circles represent the experimental (EXP.) group before $(\$$ pre $)$ and after $(\bullet$ post $)$ the intervention.

Oxygen saturation is a surveillance parameter that characterizes exercise performance on the 6MWT and helps determine the degree of disability linked to a disease during physical effort [21]. We found that the EXP. group had a greater desaturation compared to the CTL. group post $6 \mathrm{MWT}$. Some studies have shown that during the 6MWT there was an oxygen desaturation in patients with COPD, while others have shown an improvement in this parameter [22,23]. It is difficult to understand why in this study the EXP. group had a higher desaturation; however, this might represent a better anaerobic effort, indicating an improvement in lower leg muscle endurance and represents the effect of the resistance training component in the EXP. group.

In the current study, the EXP. group showed an increase in the HHb. The EXP. group intra-group analysis nearly reached statistical significance $(p=0.07)$ with an effect size of 0.14 (small effect), but not for the intergroup $(p=0.38)$. A possible explanation for the lack of an effect is that only one site was measured and that more than one point on the quadriceps may have revealed an effect. Nonetheless, a study by Koga, et al. showed results similar to ours where the $\mathrm{HHb}$ measured on 10 different points of the quadriceps during exercise had intra-group muscle deoxygenation variation, but no inter-group differences [24].

We also assessed $\mathrm{O}_{2} \mathrm{Hb}, \mathrm{tHb}$, and TSI that exhibited a consistent tendency to decrease in both EXP. and CTL. groups during the post-intervention $6 \mathrm{MWT}$, but these changes were not statistically significant. Similar results have been reported using different types of exercise trainings, such as endurance training, tapering, or strength training $[18,25,26]$.

Regarding HbDiff., there was no significant difference between groups, but it is important to note that the EXP. group had a significant increase compared to the CTL. group ( $p=0.004)$, suggesting that the concurrent aerobic-resistance training modality could have a better effect on oxygen extraction in the muscle (ES $=0.82$ ). The HbDiff. is a parameter that describes the difference between oxy- $\mathrm{Hb}$ and deoxy- $\mathrm{Hb}$ concentration $\left(\mathrm{OO}_{2} \mathrm{Hb}\right.$ $\Delta \mathrm{HHb})$. As the exercise effort increased, the difference was greater, indicating a higher $\mathrm{O}_{2}$ extraction in the muscle. Interesting to note, as shown in Figure 3, is the distance traveled by the EXP. group in the 6MWT was significantly correlated to the $\Delta \mathrm{HbDiff}$, possibly indicating an improved muscle oxygen extraction following a concurrent aerobic-resistance interval exercise program.

\section{Limitations}

There were some limitations to our study. One of the limitations is the small sample size, which included fifteen participants distributed over two groups: 1) Experimental $(n=9)$; and 2 ) Control $(n=6)$. Another limitation is the length of intervention. A 3-week intervention remains short to observe significant changes in participants. Further studies with a larger sample size and longer intervention would be beneficial in the future.

\section{Conclusions}

In conclusion, the current study shows that a concurrent aerobic-resistance interval exercise program is well tolerated by COPD patients and produces changes in vastus lateralis skeletal muscle oxygenation as well as exercise capacity. A significant association was found between HbDiff and exercise capacity (6MWT). We suggest that the increase in HbDiff appears to be the 
result of improved local muscle oxygen consumption resulting from a concurrent aerobic- resistance interval exercise program. Another important observation is the improved efficiency in obtaining significant gains in the $6 \mathrm{MWT}$ in only three weeks (half the time) in contrast to other approaches that typically span over 6 to 8 weeks.

\section{References}

1. Mador MJ, Bozkanat E, Kufel TJ (2003) Quadriceps fatigue after cycle exercise in patients with COPD compared with healthy control subjects. Chest 123: 1104-1111.

2. O'Donnell DE, Hernandez P, Kaplan A, Aaron S, Bourbeau J, et al. (2008) Canadian Thoracic Society recommendations for management of chronic obstructive pulmonary disease - 2008 update - highlights for primary care. Can Respir J 15: $1 \mathrm{~A}-8 \mathrm{~A}$.

3. Radom-Aizik S, Kaminski N, Hayek S, Halkin H, Cooper $\mathrm{DM}$, et al. (2007) Effects of exercise training on quadriceps muscle gene expression in chronic obstructive pulmonary disease. J Appl Physiol 102: 1976-1984.

4. Gloeckl R, Marinov B, Pitta F (2013) Practical recommendations for exercise training in patients with COPD. Eur Respir Rev 22: 178-186.

5. Troosters T, Casaburi R, Gosselink R, Decramer M (2005) Pulmonary rehabilitation in chronic obstructive pulmonary disease. Am J Respir Crit Care Med 172: 19-38.

6. Coppoolse R, Schols AM, Baarends EM, Mostert R, Akkermans MA, et al. (1999) Interval versus continuous training in patients with severe COPD: A randomized clinical trial. Eur Respir J 14: 258-263.

7. Borel B, Provencher S, Saey D, Maltais F (2013) Responsiveness of Various Exercise-Testing Protocols to Therapeutic Interventions in COPD. Pulm Med 2013: 410748.

8. Sabapathy S, Kingsley RA, Schneider DA, Adams L, Morris NR (2004) Continuous and intermittent exercise responses in individuals with chronic obstructive pulmonary disease. Thorax 59: 1026-1031.

9. Spruit MA, Burtin C, De Boever P, Langer D, Vogiatzis I, et al. (2016) COPD and exercise: does it make a difference? Breathe (Sheffield, England) 12: 38-49.

10. Fullenkamp AM, Laurent CM, Campbell BM (2015) Automated gait temporal-spatial assessment from nonmotorized treadmill belt speed data. Gait and Posture 41 : 141-145.

11. Gonzalez AM, Wells AJ, Hoffman JR, Stout JR, Fragala MS, et al. (2013) Reliability of the Woodway Curve (TM) NonMotorized Treadmill for Assessing Anaerobic Performance. J Sports Sci Med 12: 104-108.

12. Rabe KF, Hurd S, Anzueto A, Barnes PJ, Buist SA, et al. (2007) Global strategy for the diagnosis, management, and prevention of chronic obstructive pulmonary disease: GOLD executive summary. Am J Respir Crit Care Med 176: 532-555.
13. ATS (2002) Statement: Guidelines for the six-minute walk test. Am J Respir Crit Care Med 166: 111-117.

14. Ferrari M, Mottola L, Quaresima V (2004) Principles, techniques, and limitations of near infrared spectroscopy. Can J Appl Physiol 29: 463-487.

15. Grassi, B, Quaresima V (2016) Near-infrared spectroscopy and skeletal muscle oxidative function in vivo in health and disease: A review from an exercise physiology perspective. J Biomed Opt 21: 091313.

16. Okamoto T, Kanazawa H, Hirata K, Yoshikawa J (2003) Evaluation of oxygen uptake kinetics and oxygen kinetics of peripheral skeletal muscle during recovery from exercise in patients with chronic obstructive pulmonary disease. Clin Physiol Funct Imaging 23: 257-262.

17. Puente-Maestu L, Tena T, Trascasa C, Perez-Parra J, Godoy R, et al. (2003) Training improves muscle oxidative capacity and oxygenation recovery kinetics in patients with chronic obstructive pulmonary disease. Eur J Appl Physiol 88: 580-587.

18. Tabira K, Horie J, Fujii H, Aida T, Ito K, et al. (2012) The relationship between skeletal muscle oxygenation and systemic oxygen uptake during exercise in subjects with COPD: a preliminary study. Respir Care 57: 1602-1610.

19. Vogiatzis I, Athanasopoulos D, Stratakos G, Garagouni C, Koutsoukou A, et al. (2009) Exercise-induced skeletal muscle deoxygenation in O- supplemented COPD patients. Scand J Med Sci Sports 19: 364-372.

20. Bohannon RW, Crouch R (2016) Minimal clinically important difference for change in 6-minute walk test distance of adults with pathology: a systematic review. J Eval Clin Pract 23: 377-381.

21. Moreira MA, Medeiros GA, Boeno FP, Sanches PR, Silva Junior DP, et al. (2014) Oxygen desaturation during the six-minute walk test in COPD patients. J Bras Pneumol 40: 222-228.

22. Ozalevli S, Ozden A, Itil O, Akkoclu A (2007) Comparison of the Sit-to-Stand Test with 6 min walk test in patients with chronic obstructive pulmonary disease. Respir Med 101: 286-293.

23. Poulain M, Durand F, Palomba B, Ceugniet F, Desplan J, et al. (2003) 6-minute walk testing is more sensitive than maximal incremental cycle testing for detecting oxygen desaturation in patients with COPD. Chest 123: 1401-1407.

24. Koga S, Poole DC, Ferreira LF, Whipp BJ, Kondo N, et al. (2007) Spatial heterogeneity of quadriceps muscle deoxygenation kinetics during cycle exercise. J Appl Physiol 103 : 2049-2056.

25. Neary JP, McKenzie DC, Bhambhani YN (2005) Muscle oxygenation trends after tapering in trained cyclists. Dyn Med 4: 4.

26. Odagawa J, Sekikawa K, Kawaguchi K, Takahashi M, Morita N (2009) Influence of home-based pulmonary rehabilitation on muscle oxygenation in elderly patients with chronic obstructive pulmonary disease. J Physiother Sci 21. 